\title{
Simplified State Observer of Hyperchaotic Lü Attractor with One Voltage-sensed Signal
}

\author{
Chi-Hsin Yang, Hui-Cun Shen, Kun-Chieh Wang, ${ }^{*}$ and Hong-Yi Chen \\ Zhaoqing University, Zhaoqing City, Guangdong Province 526061, P.R. China \\ (Received November 23, 2018; accepted January 18, 2019)
}

Keywords: simplified state observer, voltage measurement, hyperchaotic Lü attractor, exponential stability

The main goal of this study is to develop a simplified state observer for a four-dimensional hyperchaotic Lü attractor implemented by a circuit system. The elements of the state vector can be fully reconstructed by feeding the proposed state observer with only one continuously sensed voltage measurement. By utilizing the time-domain approach, the estimated state errors are proven to satisfy global and exponential stability, and the exponential convergence rate can be specified via the values of system parameters. Numerical studies are also presented to illustrate the effectiveness of the proposed scheme.

\section{Introduction}

Since the introduction of a method of synchronizing two identical chaotic systems with different initial conditions in the pioneering work of Pecora and Carroll, ${ }^{(1)}$ chaos control and synchronization have become a very important topic in nonlinear science. Owing to the huge range of potential applications, chaos control and chaos synchronization ${ }^{(2-9)}$ have received a great deal of attention among researchers in many fields, such as physical ${ }^{(10)}$ and chemical systems, ${ }^{(11,12)}$ power converters, ${ }^{(13)}$ ecological and biological systems, ${ }^{(14,15)}$ financial systems, ${ }^{(16)}$ and secure communication. ${ }^{(17,18)}$

In applications, it is either inefficient or impractical to measure all the elements of the state vector under practical hardware limitations. Moreover, a state observer can be applied to compute the reconstruction and take the place of sensor signals in the event of sensor failures. For these reasons, state observer design is an essential and meaningful task for chaos control and synchronization. Numerous studies ${ }^{(19-21)}$ on the state observer design of many chaotic systems have been reported. However, the system configurations of state observers in the previous works are sometimes complicated and almost all the state variables of the measured drive system must be supplied.

In a reasonable and practical way, the configuration of the state observer should be simplified and the number of measured state inputs in the chaotic system should be small. In the literature, simple state observers with the state of one drive system as the input were 
investigated by Sun ${ }^{(22,23)}$ for three-dimensional generalized Lorenz and Chen chaotic systems. However, such a simple state observer with the feeding of one state variable of the drive system has not yet been addressed for more complex and higher-dimensional systems.

Motivated by the aforementioned studies, a simplified state observer design for the fourdimensional (4D) hyperchaotic Lü attractor implemented by a circuit system $^{(24)}$ is developed utilizing the time-domain approach. The state vector can be fully reconstructed by the proposed state observer with one continuously sensed voltage signal of the 4D chaotic circuit system. The estimated state errors are proven to guarantee global and exponential stability. Furthermore, the exponential convergence rates can also be specified accurately via the parameters of such a system. Simulations are performed to show the validity of the main results. The rest of this paper is organized as follows. In Sect. 2, a problem description is given and the main results are derived. In Sect. 3, simulations to demonstrate the effectiveness of the main results are reported. Finally, conclusions are given in Sect. 4.

\section{Problem Description and Main Results}

A 4D hyperchaotic Lü attractor introducing state feedback based on the Lü system ${ }^{(25,26)}$ was constructed in Ref. 24. In this system with the output $y(t)$ including the time series of the first state variable, $x_{1}(t)$, has the form

$$
\begin{aligned}
& \left\{\begin{array}{l}
\dot{x}_{1}=a\left(x_{2}-x_{1}\right)+x_{4} \\
\dot{x}_{2}=c x_{2}-x_{1} x_{3} \\
\dot{x}_{3}=-b x_{3}+x_{1} x_{2} \\
\dot{x}_{4}=d x_{4}+x_{1} x_{3}
\end{array}\right. \\
& y(t)=x_{1}(t)
\end{aligned}
$$

where $\boldsymbol{x}(t)=\left[\begin{array}{llll}x_{1}(t) & x_{2}(t) & x_{3}(t) & x_{4}(t)\end{array}\right]^{\mathrm{T}}$ and $a, b, c$, and $d$ are the state vector and system parameters, respectively. It has three quadratic nonlinearities and thus has more complex dynamical behaviors than normal chaotic systems. Chen et al. ${ }^{(24)}$ proved that the dynamical behaviors of the system in Eq. (1) switch among a periodic orbit, a chaotic state, and a hyperchaotic state with increasing parameter $d$. When the system parameters are $a=36, b=$ 3 , and $c=20$, it was shown that the system in Eq. (1) has a periodic orbit for $-1.03 \leq d \leq-0.46$, a chaotic attractor for $-0.46<d \leq-0.35$, and hyperchaotic attractors for $-0.35<d \leq 1.30$. The hyperchaotic Lü attractor with the system parameters $a=36, b=3, c=20$, and $d=0.5$, and the initial conditions $\left(x_{1}(0), x_{2}(0), x_{3}(0), x_{4}(0)\right)=(0.1,0.1,25,0.1)$ is depicted in Fig. 1.

The circuit design in Ref. 24 to realize the 4D hyperchaotic Lü system is shown in Fig. 2. The output voltages, $x_{i}, i=1, \cdots, 4$, in the illustrated circuit are equivalent to the state variables of the 4D system. It was concluded that the occurrence of chaotic or hyperchaotic behavior depended on the position of switch $K$ and the values of the resistors in the circuit. From the viewpoint of practical usage, it is easy to continuously sense one of the output voltages, for 

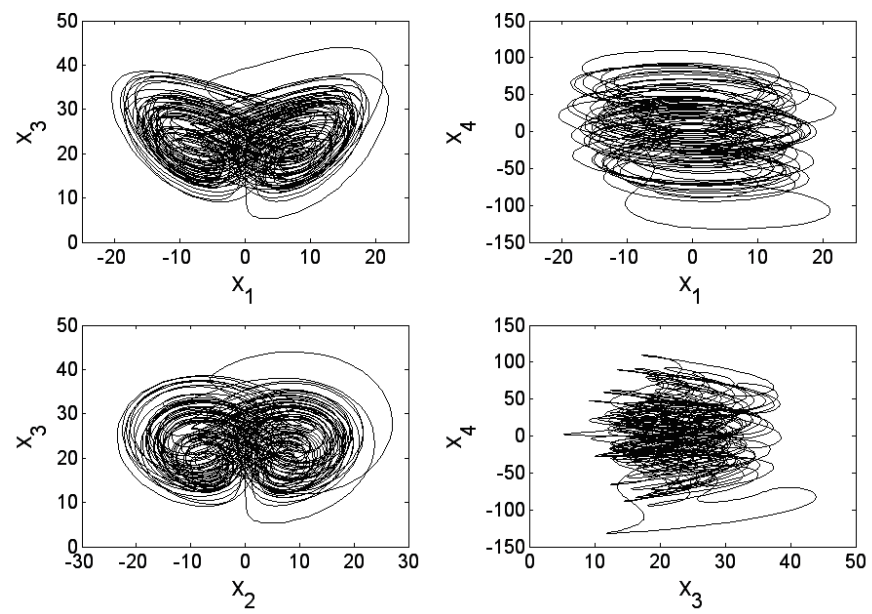

Fig. 1. 4D hyperchaotic Lü attractor with $a=36, b=3, c=20$, and $d=0.5$.

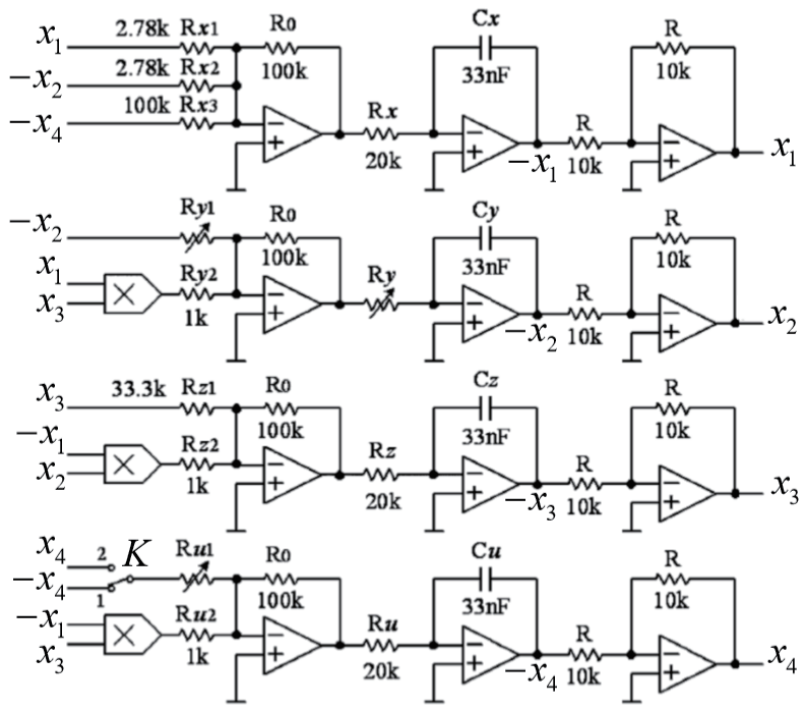

Fig. 2. Circuit implementation of 4D hyperchaotic Lü system. ${ }^{(24)}$

example, the first one $x_{1}$, and record the measurements as the time series using a suitable voltage sensing system.

In the following, a simplified state observer design utilizing the sensed time signals of the first voltage $x_{1}$ of the circuit system in Eq. (1) is developed. Before proceeding to the main results, a definition that will be applied in the main theorem is presented. For the design of a simplified state observer of the system in Eq. (1), an equivalent system representation of the system in Eq. (1) will also be introduced in a lemma.

Definition. The states of system (1) can be globally and exponentially reconstructed if the state observer $\dot{\hat{\boldsymbol{x}}}(t)=\boldsymbol{f}(\boldsymbol{x}(t), y(t))$ exists, where $\hat{\boldsymbol{x}}(t)=\left[\begin{array}{llll}\hat{x}_{1}(t) & x_{2}(t) & x_{3}(t) & x_{4}(t)\end{array}\right]^{\mathrm{T}}$ $\hat{\boldsymbol{x}}(t)=\left[\begin{array}{llll}\hat{x}_{1}(t) & x_{2}(t) & x_{3}(t) & x_{4}(t)\end{array}\right]^{\mathrm{T}}$ expresses the reconstructed state vector of system (1) and 
positive constants $A$ and $B$ such that

$$
\|\boldsymbol{e}(t)\|=\|\boldsymbol{x}(t)-\hat{\boldsymbol{x}}(t)\| \leq A \exp (-B t), \quad \forall t \geq 0
$$

for any initial condition $\hat{\boldsymbol{x}}(0)$ in a set $D_{4} \subseteq R^{4}$. In this case, the positive constant $B$ is named the guaranteed exponential convergent rate.

Lemma. The system in Eq. (1) can be transformed into the following equivalent system representation:

$$
\left\{\begin{array}{l}
\dot{\sigma}(t)=-\frac{c-a d}{a-1} \sigma(t)+\frac{c-d}{a-1}\left(\dot{x}_{1}(t)+a x_{1}(t)\right) \\
x_{2}(t)=\frac{1}{a-1}\left(\dot{x}_{1}(t)+a x_{1}(t)-\sigma(t)\right) \\
x_{4}(t)=\frac{-1}{a-1}\left(\dot{x}_{1}(t)+a x_{1}(t)\right)+\frac{a}{a-1} \sigma(t) \\
\dot{x}_{3}(t)=-b x_{3}(t)+x_{1}(t) x_{2}(t)
\end{array}\right.
$$

where the auxiliary state variable $\sigma(t)$ is defined as

$$
\sigma(t)=x_{2}(t)+x_{4}(t)
$$

Proof. The first equation in the system (1) can be expressed as

$$
a x_{2}+x_{4}=\dot{x}_{1}+a x_{1}
$$

Then, $x_{2}(t)$ and $x_{4}(t)$ can be solved from Eqs. (3) and (4) and expressed as

$$
\begin{aligned}
& x_{2}(t)=\frac{1}{a-1}\left(\dot{x}_{1}(t)+a x_{1}(t)-\sigma(t)\right) \\
& x_{4}(t)=\frac{-1}{a-1}\left(\dot{x}_{1}(t)+a x_{1}(t)\right)+\frac{a}{a-1} \sigma(t) .
\end{aligned}
$$

Substituting the second and fourth equations in the system in Eq. (1) into the time derivative of the auxiliary state variable defined in Eq. (3) yields

$$
\dot{\sigma}(t)=\dot{x}_{2}(t)+\dot{x}_{4}(t)=c x_{2}(t)+d x_{4}(t)
$$

The first-order differential equation of the auxiliary state variable $\sigma(t)$ is obtained by substituting Eq. (5) into Eq. (6). 


$$
\dot{\sigma}(t)=-\frac{c-a d}{a-1} \sigma(t)+\frac{c-d}{a-1}\left(\dot{x}_{1}(t)+a x_{1}(t)\right)
$$

This completes the proof of Lemma.

Theorem. The states of system (1) can be globally and exponentially reconstructed in the case of $d<c / a$. A suitable state observer is given by

$$
\left\{\begin{array}{l}
\hat{x}_{1}(t)=y(t) \\
\hat{x}_{2}(t)=\frac{1}{a-1}(\dot{y}(t)+a y(t)-\hat{\sigma}(t)) \\
\hat{x}_{4}(t)=\frac{-1}{a-1}(\dot{y}(t)+a y(t))+\frac{a}{a-1} \hat{\sigma}(t) \\
\dot{\hat{x}}_{3}(t)=-b \hat{x}_{3}(t)+\hat{x}_{1}(t) \hat{x}_{2}(t)
\end{array}\right.
$$

where $\hat{\sigma}(t)$ is the reconstructed auxiliary state variable and can be evaluated using the following equation:

$$
\dot{\hat{\sigma}}(t)=-k \hat{\sigma}(t)+\frac{c-d}{a-1}(\dot{y}(t)+a y(t)),
$$

with $k=\frac{c-a d}{a-1}>0$.

Proof. Consider the state errors between the state variables of systems in Eqs. (1) and (8) defined by $e_{i}(t)=x_{i}(t)-\hat{x}_{i}(t), \forall i \in\{1,2,3,4\}$. It can be readily found that

$$
e_{1}(t)=x_{1}(t)-\hat{x}_{1}(t)=0, \forall t \geq 0 \text {. }
$$

To obtain $e_{2}(t)=0$ and $e_{4}(t)=0$, two linear equations containing $e_{2}(t)$ and $e_{4}(t)$ should be proposed.

$$
\begin{aligned}
a e_{2}(t) & +e_{4}(t)=a\left(x_{2}(t)-\hat{x}_{2}(t)\right)+\left(x_{4}(t)-x_{4}(t)\right) \\
= & a x_{2}(t)+x_{4}(t)-a\left[\frac{1}{a-1}(\dot{y}(t)+a y(t)-\hat{\sigma}(t))\right] \\
& \quad-\left[\frac{-1}{a-1}(\dot{y}(t)+a y(t))+\frac{a}{a-1} \hat{\sigma}(t)\right] \\
= & \dot{x}_{1}(t)+a x_{1}(t)-(\dot{y}(t)+a y(t))=0
\end{aligned}
$$




$$
\begin{aligned}
\dot{e}_{\sigma}(t) & =\dot{\sigma}(t)-\dot{\hat{\sigma}}(t) \\
& =-\frac{c-a d}{a-1} \sigma(t)+\frac{c-d}{a-1}\left(\dot{x}_{1}(t)+a x_{1}(t)\right)-k \hat{\sigma}(t)+\frac{c-d}{a-1}(\dot{y}(t)+a y(t)) \\
& =-k e_{\sigma}(t) \\
\Rightarrow e_{\sigma}(t) & =e_{2}(t)+e_{4}(t)=e_{\sigma}(0) \exp (-k t), \forall t \geq 0
\end{aligned}
$$

The stability of $e_{\sigma}(t)$ is guaranteed by the fact that $k=\frac{c-a d}{a-1}>0$, that is, $d<c / a$. It can be concluded that

$$
\left|e_{2}(t)+e_{4}(t)\right|=\left|e_{\sigma}(0)\right| \exp (-k t)=0, \forall t \geq t_{\text {settling }}>0
$$

where $t_{\text {settling }}>0$ is the settling time for $\left|e_{\sigma}(t)\right|$ approaching zero. Thus, $e_{2}(t)=0$ and $e_{4}(t)=0$ are obtained by solving Eqs. (11) and (12). Consequently, the stability of the estimated error $e_{3}(t)$ is derived as

$$
\begin{aligned}
\dot{e}_{3}(t) & =\dot{x}_{3}(t)-\dot{\hat{x}}_{3}(t) \\
& =-b x_{3}(t)+x_{1}(t) x_{2}(t)+b \hat{x}_{3}(t)-\hat{x}_{1}(t) \hat{x}_{2}(t) \\
& =-b\left[x_{3}(t)-\hat{x}_{3}(t)\right]+x_{1}(t)\left[x_{2}(t)-\hat{x}_{2}(t)\right] \\
& =-b e_{3}(t), \quad \forall t \geq t_{\text {settling }}>0 .
\end{aligned}
$$

This implies that

$$
\begin{aligned}
& \frac{d}{d t}\left[e_{3}(t) \exp (b t)\right]=0 \\
& \Rightarrow e_{3}(t)=e_{3}(0) \exp (-b t) \\
& \Rightarrow\left|e_{3}(t)\right|=\left|e_{3}(0)\right| \exp (-b t), \quad \forall t \geq t_{\text {settling }}>0 .
\end{aligned}
$$

Equations (10) to (13) yield

$$
\|\boldsymbol{e}(t)\|=\sqrt{e_{1}^{2}(t)+e_{2}^{2}(t)+e_{3}^{2}(t)+e_{4}^{2}(t)} \leq\left|e_{3}(0)\right| \exp (-b t), \quad \forall t \geq 0 .
$$

According to Definition, the states of the system in Eq. (1) can be globally and exponentially reconstructed by the state observer in Eq. (8) associated with Eq. (9). This completes the proof.

\section{Numerical Studies}

Consider the 4D hyperchaotic Lü attractor in Eq. (1) with $a=36, b=3, c=20$, and $d \in(-1.03,1.3]$. Assume that the time signals of the first voltage $x_{1}$ of the circuit system are 
produced by performing a suitably practical measurement. From Theorem, it is concluded that the system in Eq. (1) can be globally and exponentially reconstructed by the following simplified state observer in the case of $-1.03 \leq d<c / a=5 / 9$. (1) can be globally and exponentially reconstructed by the following simplified state observer:

$$
\left\{\begin{array}{l}
\hat{x}_{1}(t)=y(t) \\
\hat{x}_{2}(t)=\frac{1}{35}(\dot{y}(t)+36 y(t)-\hat{\sigma}(t)) \\
\left.\hat{x}_{4}(t)=\frac{-1}{35}(\dot{y}(t)+36 y(t))+\frac{36}{35} \hat{\sigma}(t)\right) \\
\dot{\hat{x}}_{3}(t)=-3 \hat{x}_{3}(t)+\hat{x}_{1}(t) \hat{x}_{2}(t)
\end{array}\right.
$$

In Eq. (14), $\hat{\sigma}(t)$ is the reconstructed auxiliary state variable and can be evaluated as

$$
\dot{\hat{\sigma}}(t)=-\frac{20-36 d}{35} \hat{\sigma}(t)+\frac{20-d}{35}(\dot{y}(t)+36 y(t)) .
$$

The guaranteed exponential convergence rates are $B_{1}=\frac{20-36 d}{35}$ and $B_{2}=3$ for the reconstructed auxiliary state variable $\hat{\sigma}(t)$ and the reconstructed state variable $\hat{x}_{3}(t)$, respectively. In the following, simulations on two cases of 4D hyperchaotic Lü attractors are performed to illustrate the efficiency of the proposed simple state observer.

The numerical simulations are carried out using MATLAB/SIMULINK software. The fourth-order Runge-Kutta method with a time step of 0.0001 is used to solve the system. The initial conditions of the 4D hyperchaotic Lü attractor (1) and proposed simplified state observer are selected to be $x_{1}(0)=y(0)=10, x_{2}(0)=10, x_{3}(0)=10, x_{4}(0)=10$, and $\hat{\sigma}(0)=0, \hat{x}_{3}(0)=0$, respectively. From Eqs. (1) and (8), the following initial values are evaluated: $\dot{y}(0)=10$, $\hat{x}_{2}(0)=10.57, \hat{x}_{4}(0)=-10.57$. The time responses of the estimated state errors between the

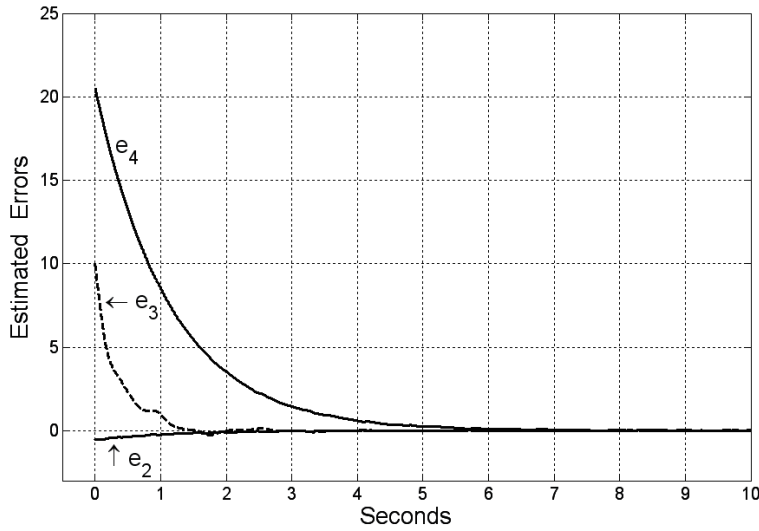

Fig. 3. Time responses of estimated errors for $d=$ -0.3 .

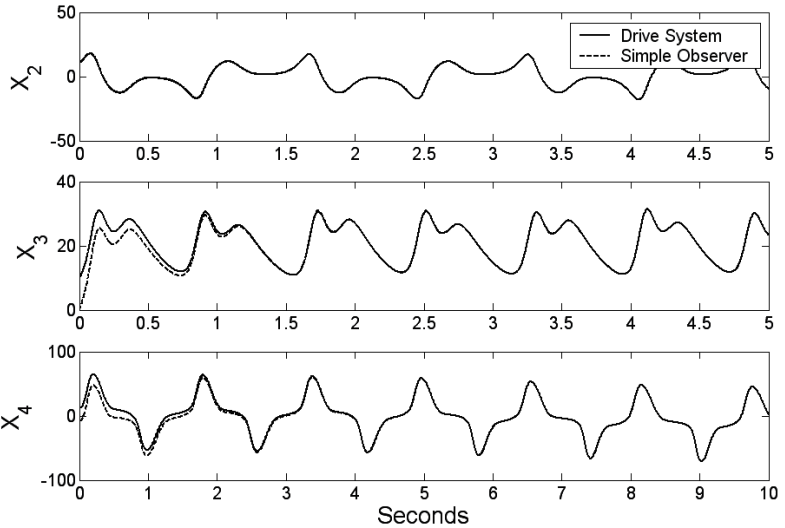

Fig. 4. Time responses of the system in Eq. (1) and state observer in Eq. (14) associated with Eq. (15) for $d=-0.3$. 


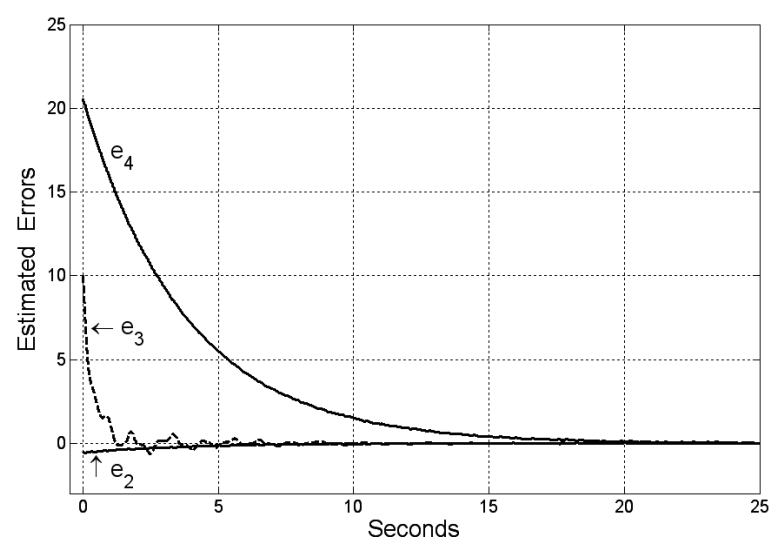

Fig. 5. Time responses of estimated errors for $d=0.3$.

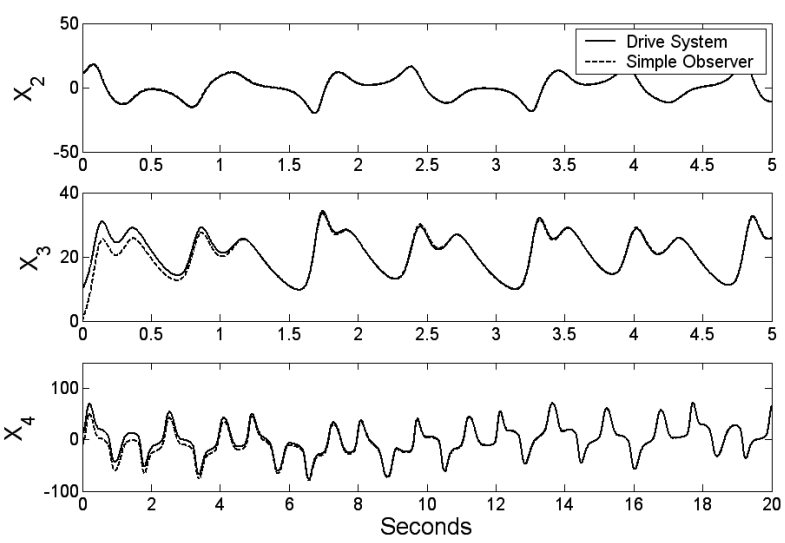

Fig. 6. Time responses of the system in Eq. (1) and state observer in Eq. (14) associated with Eq. (15) for $d=0.3$.

simplified state observer (14) associated with Eq. (15) and the system in Eq. (1) for the case of $d=-0.3$ are depicted in Fig. 3. In this case, the guaranteed exponential convergence rate is $B_{1}=0.88$. Time responses of the system in Eq. (1) and the simplified state observer in Eq. (14) associated with Eq. (15) are shown in Fig. 4. It is shown that the reconstructed states of Eq. (14) associated with Eq. (15) are readily convergent to the states of the system in Eq. (1). In the case of $d=0.3$, the guaranteed exponential convergence rate is $B_{1}=0.263$. The time responses of the reconstructed errors and state responses are illustrated in Figs. 5 and 6, respectively. From the above simulation results, it is shown that the system in Eq. (1) can be globally and exponentially reconstructed using the proposed simplified state observer in Eq. (14) associated with Eq. (15).

\section{Conclusions}

In this study, the 4D hyperchaotic Lü attractor implemented by the circuit system proposed by Chen et al. ${ }^{(24)}$ is considered and the state reconstruction problem of this system is studied. Utilizing the time domain approach, a simplified state observer applied to one continuously measured voltage signal of the 4D hyperchaotic circuit system is designed, and state errors are proven to satisfy global and exponential stability. The guaranteed exponential convergence rate can be estimated from the system parameters provided that $d<c / a$ is satisfied. Finally, simulations are performed to demonstrate the effectiveness of the proposed scheme.

\section{References}

1 L. M. Pecora and T. L. Carroll: Phys. Rev. Lett. 64 (1990) 821. https://doi.org/10.1103/PhysRevLett.64.821

2 A. Loría: IEEE Trans. Circuits Syst. II 56 (2009) 674. 10.1109/TCSII.2009.2025624

3 C. C. Yang: Appl. Math. Comput. 216 (2010) 1316. https://doi.org/10.1016/j.amc.2010.02.026

4 C. C. Yang: Nonlinear Dyn. 63 (2011) 447. https://doi.org/10.1007/s11071-010-9814-3

5 C. C. Yang: J. Franklin Inst. 349 (2012) 349. https://doi.org/10.1016/j.jfranklin.2011.11.013

6 C. C. Yang: Appl. Math. Comput. 219 (2013) 5152. https://dx.doi.org/10.1016/j.amc.2012.11.003

7 C. C. Yang: Arabian J. Sci. Eng. 39 (2014) 2413. https://10.1007/s13369-013-0768-x 
8 R. Luo and Y. Zeng: Nonlinear Dyn. 80 (2015) 989. https://doi.org/10.1007/s11071-015-1923-6

9 J. Yu, J. Le, and D. Liu: Optik 130 (2017) 1053. https://doi.org/10.1016/j.ijleo.2016.11.108

10 K. S. Sudheer and M. Sabir: Phys. Lett. A 373 (2009) 1847. https://doi.org/10.1016/j.physleta.2009.03.052

11 T. P. Chang: Sens. Mater. 30 (2018) 1911. https://dx.doi.org/10.18494/SAM.2018.2012

12 C. J. Xu and Y. Wu: Appl. Math. Modell. 39 (2015) 2295. https://doi.org/10.1016/j.apm.2014.10.030

13 V. Avrutin, E. Mosekilde, Z. T. Zhusubaliyev, and L. Gardini: Chaos 25 (2015) 043114. https://dx.doi. org/10.1063/1.4918299

14 S. K. Dana, P. K. Roy, and J. Kurths: Complex Dynamics in Physiological Systems: From Heart to Brain (Springer, New York, 2009) p. 127. https://dx.doi.org 10.1007/978-1-4020-9143-8

15 B. Blasius and L. Stone: Int. J. Bifurcation Chaos 10 (2000) 2361. https://doi.org/10.1142/S0218127400001511

16 C. Lin: J. Control Sci. Eng. 2018 (2018) 4976380. https://doi.org/10.1155/2018/4976380

17 R. Martfnez-Guerra and Y. Wen: Int. J. Bifurcation Chaos 18 (2008) 235. https://doi.org/10.1142/ S0218127408020264

18 W. D. Chang, S. P. Shih, and C. Y. Chen: J. Control Sci. Eng. 2015 (2015) 471913. https://dx.doi. org $/ 10.1155 / 2015 / 471913$

19 C. Hua and X. Guan: Phys. Lett. A 334 (2005) 382. https://doi.org/10.1016/j.physleta.2004.11.050

20 R. Martfnez-Guerra, W. Yu, and E. Cisneros-Saldana: Chaos Solitons Fractals 36 (2008) 1141. https://doi. org/10.1016/j.chaos.2006.07.039

21 J. Zheng and B. Cui: Chaos Solitons Fractals 112 (2018) 141. https://doi.org/10.1016/j.chaos.2018.04.038

22 Y. J. Sun: Chaos Solitons Fractals 39 (2009) 1641. https://doi.org/10.1016/j.chaos.2007.06.043

23 Y. J. Sun: Phys. Lett. A 374 (2010) 933. https://doi.org/10.1016/j.physleta.2009.12.019

24 A. Chen, J. Lu, and S. Yu: Physica A 364 (2006) 103. https://doi.org/10.1016/j.physa.2005.09.039

25 J. Lu and G. Chen: Int. J. Bifurcation Chaos 12 (2002) 659. https://doi.org/10.1142/S0218127402004620

26 J. Lu, G. Chen, and S. Zhang: Int. J. Bifurcation Chaos 12 (2002) 1001. https://doi.org/10.1142/ S0218127402004851 